\title{
Aneuploidy in Cancer and Aging
}

\section{Ryan M. Naylor ${ }^{1}$ and Jan M. van Deursen ${ }^{1,2}$}

${ }^{1}$ Department of Biochemistry and Molecular Biology, ${ }^{2}$ Department of Pediatric and Adolescent Medicine, Mayo Clinic, Rochester, Minnesota 55905; email: vandeursen.jan@mayo.edu

Annu. Rev. Genet. 2016. 50:45-66

The Annual Review of Genetics is online at genet.annualreviews.org

This article's doi:

10.1146/annurev-genet-120215-035303

Copyright (c) 2016 by Annual Reviews. All rights reserved

\section{Keywords}

chromosomal instability, anaphase-promoting complex/cyclosome

\section{Abstract}

Chromosomal instability (CIN), the persistent inability of a cell to faithfully segregate its genome, is a feature of many cancer cells. It stands to reason that CIN enables the acquisition of multiple cancer hallmarks; however, there is a growing body of evidence suggesting that CIN impairs cellular fitness and prevents neoplastic transformation. Here, we suggest a new perspective to reconcile this apparent paradox and share an unexpected link between aneuploidy and aging that was discovered through attempts to investigate the CIN-cancer relationship. Additionally, we provide a comprehensive overview of the function and regulation of the anaphase-promoting complex, an E3 ubiquitin ligase that mediates high-fidelity chromosome segregation, and describe the mechanisms that lead to whole-chromosome gain or loss. With this review, we aim to expand our understanding of the role of CIN in cancer and aging with the long-term objective of harnessing this information for the advancement of patient care. 


\section{ANEUPLOIDY AND CANCER: A HISTORICAL CONTEXT}

Tumorigenesis is a multistep process in which normal cells progress toward a transformed state through the acquisition of cancer hallmarks (46). Among these hallmarks are the abilities to sustain proliferative signaling, evade cell cycle inhibition, and resist cell death (46). In the early 1900s, Theodor Boveri postulated that aneuploidy, i.e., any numerical karyotype abnormality, might be the driving force behind cellular transformation and tumorigenesis (10). Indeed, in their 2011 landmark paper, Hanahan \& Weinberg (46) solidified genome instability as an enabling characteristic of cancer. The rationale behind this designation is that genome instability can lead to the acquisition of a mutator phenotype, which in turn increases the probability that a cell will acquire cancer hallmarks. However, more than 100 years after Boveri's hypothesis, the precise relationship between aneuploidy and tumorigenesis remains the subject of much debate $(39,101$, $103,110,138)$. Fueling this debate are growing bodies of seemingly conflicting evidence both in favor of and opposing the idea that aneuploidy is a driver of tumorigenesis. Favoring Boveri are the observations that the vast majority of malignant neoplasms are aneuploid, that multiple aneuploid mouse models are predisposed to cancer (110), and that aneuploidy drives tumor suppressor loss of heterozygosity in some sensitized backgrounds (4). Arguing against Boveri are the observations that aneuploidy delays spontaneous immortalization (140) and elicits a variety of tumor-suppressive stress responses, including growth and cell cycle arrest (126, 140), apoptosis $(61,70,78,115)$, mitotic catastrophe (130), and senescence (77). Moreover, it is well documented that several aneuploid mouse models are not cancer prone (110). Thus, whether aneuploidy is a cause or consequence of transformation and whether aneuploidy promotes or suppresses tumorigenesis remain important unanswered questions despite Boveri's long-standing hypothesis.

The overall objective of this article is to provide a new perspective on this century-old question and distill what appear to be contradictory observations into a more holistic, unified understanding of the role of aneuploidy in cancer. Furthermore, we aim to deliver a compelling vignette as to how an unexpected link between aneuploidy and aging was accidentally discovered through attempts to test Boveri's hypothesis. To do this, we first provide an overview of the function and regulation of the anaphase-promoting complex/cyclosome (APC/C), which is the central mediator of mitosis, and then describe the mechanisms by which aneuploidy arises de novo. We conclude by briefly describing the aneuploidy-cancer paradox before offering a new perspective on this important issue, and, finally, we consider a recently discovered link between whole-chromosomal instability (W-CIN), aneuploidy, and aging.

\section{THE ANAPHASE-PROMOTING COMPLEX/CYCLOSOME}

The goal of the mammalian cell cycle is to faithfully replicate the cell's DNA and segregate it evenly between two daughters. The climactic segregation of chromosomes in anaphase is a highly complex and regulated process mediated by the APC/C. In the following section, we discuss the basic biology of the APC/C and consider the multiple mechanisms of APC/C regulation the cell uses to ensure that both daughters receive a full, unadulterated complement of chromosomal content.

\section{Structure and Function of the Anaphase-Promoting Complex/Cyclosome}

The APC/C is an E3 ubiquitin ligase that mediates separation of sister chromatids, cytokinesis, maintenance of G1 phase, and initiation of S phase by polyubiquitinating various substrates and targeting them for degradation by the $26 \mathrm{~S}$ proteasome (118). Ubiquitin is a small, ubiquitously 
expressed peptide that modifies other proteins through a well-defined, three-step enzymatic reaction (reviewed in 67). Briefly, an E1 ubiquitin-activating enzyme forms a thioester bond with ubiquitin in an ATP-dependent fashion. The ubiquitin molecule is then transferred to a cysteine residue on an E2 ubiquitin-conjugating enzyme. Finally, ubiquitin is conjugated via covalent amide bond formation to a lysine $(\mathrm{K})$ residue within the substrate, a reaction that is catalyzed by an E3 ubiquitin ligase. This final step occurs in one of two ways: either the E3 ubiquitin ligase simultaneously binds the E2 ubiquitin protein complex and substrate in order to facilitate direct ubiquitin transfer, as is the case for RING E3 ligases like the APC/C $(66,67)$ (Figure 1), or the E3 ligase can receive a ubiquitin molecule from an E2 enzyme at an active-site cysteine prior to modifying the substrate, as is the case for HECT E3 ligases. Propagation of the ubiquitin chain takes place in much the same way as the initiating ubiquitin-substrate conjugation step. The main difference is that the E3 ligase generates an amide bond between the C-terminal glycine of the incoming ubiquitin and the $\varepsilon$-amino group of the ubiquitin molecule that is already attached to the substrate or polyubiquitin chain. As it relates to APC/C, Ubch10 is the E2 enzyme that initiates ubiquitin-substrate conjugation $(11,141)$ and Ube2s elongates ubiquitin chains (34). Polyubiquitin chains are traditionally designated by their linkage patterns. The APC/C is thought to primarily generate K11-linked chains $(64,91)$, a pattern associated with proteasomal degradation, but mixed chains containing K11, K48, and K63 linkages have also been reported (93).

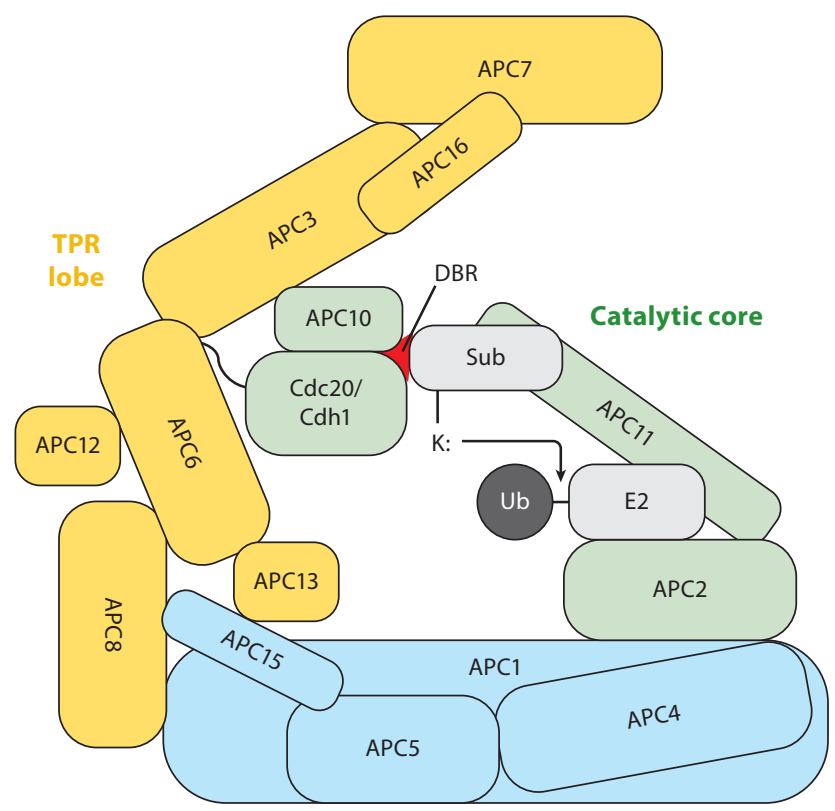

Platform

Figure 1

Structure and function of the anaphase-promoting complex/cyclosome (APC/C). The APC/C is a multimeric, cullin-RING E3 ubiquitin (Ub) ligase. It is organized into three domains: the platform (blue), the tetratricopeptide repeat (TPR) lobe (yellow), and the catalytic core (green). The coactivating proteins Cdc20 and Cdh1 form a bipartite destruction-box (D-box) receptor (DBR; red) together with the Apc10 subunit. Cullin (Apc2) and RING (Apc11) simultaneously bind the E2 ubiquitin complex and the substrate (sub) to facilitate a nucleophilic substitution reaction between the amino group of a lysine (K) residue on the substrate and the thioester bond of the E2-Ub intermediate. Adapted with permission from Macmillan Publishers Ltd: Nat. Rev. Mol. Cell Biol. 16, 82-94 (2015), Reference 118. 
The multimeric APC/C is a member of the cullin-RING ligase family, and its activity is dependent on coactivation by $\mathrm{Cdc} 20$ or Cdh1 (12). Structurally, the APC/C is organized into three domains: the platform, the tetratricopeptide repeat (TPR) lobe, and the catalytic core $(12,118)$ (Figure 1). The catalytic core is what gives the APC/C its enzymatic function. It is made up of Apc2 (cullin), Apc10, Apc11 (RING), and the APC/C coactivator Cdc20 or Cdh1. Together, Apc10 and Cdc20 or Cdh1 recruit and recognize substrates by forming a bipartite coreceptor that recognizes destruction-box (D-box) degrons on candidate substrates (12, 13, 23, 50, 68, 71) (Figure 1). The catalytic subunits, Apc2 and Apc11, are perfectly situated just below the bipartite receptor in order to facilitate Ubch10-mediated ubiquitin initiation and Ube2s-mediated chain elongation $(11,12)$ (Figure 1).

\section{Cdc20 and Cdh1: Coactivators of the Anaphase-Promoting Complex/Cyclosome}

$\mathrm{Cdc} 20$ and $\mathrm{Cdh} 1$ are structurally similar APC/C coactivating proteins that associate with the $\mathrm{APC} / \mathrm{C}$ at various points throughout the cell cycle. The $\mathrm{C}$-terminal WD40 $\beta$-propeller domains of Cdc20 and Cdh1 interact with substrates containing KEN-box degrons and, as discussed above, cooperate with Apc10 to form bipartite D-box coreceptors. These coactivating proteins are known to interact with the APC/C through at least two distinct mechanisms, both of which are necessary for APC/C binding and coactivation. First, the C-box domain is required for coactivator-APC/C interaction through an unknown mechanism and for stimulating $\mathrm{APC} / \mathrm{C}$ catalytic activity (73). Second, the isoleucine-arginine (IR) tail is known to bind to the TPR proteins Apc3 and Apc6 (147) (Figure 1). Notably, a pro-drug version of tosyl-L-arginine methyl ester (proTAME) antagonizes the APC/C by interfering with the IR tail-dependent interaction of Cdc20 and Cdh1 with the APC/C (147).

\section{Regulating Anaphase-Promoting Complex/Cyclosome ${ }^{\mathrm{Cdc20}}$ and Anaphase-Promoting Complex/Cyclosome ${ }^{\text {Cdh1 Activity }}$}

Progression through mitosis and G1 requires the sequential activity of APC/C $\mathrm{Cdc}^{\mathrm{Cd} 2}$ followed by $\operatorname{APC/C} \mathrm{C}^{\mathrm{Cdh} 1}(96,97)$. Traditionally, APC/C $\mathrm{C}^{\mathrm{Cdc} 20}$ is thought to be active beginning in prometaphase and lasts until anaphase onset, at which point Cdh1 replaces $\mathrm{Cdc} 20$ as the predominant APC/C coactivator. APC/C $C^{C d h 1}$ remains active from anaphase through the subsequent $\mathrm{G} 1 / \mathrm{S}$ transition. Maintaining this sequence of events requires precise regulation of the APC/C and its coactivating subunits. The complex regulatory mechanisms that determine how the cell synchronizes the activity of the $\mathrm{APC} / \mathrm{C}$ and its coactivators are considered here.

Mitotic checkpoint complex. The mitotic checkpoint complex (MCC) is undoubtedly the most studied and well-known APC/C inhibitor. The MCC mediates the spindle assembly checkpoint (SAC), which inhibits progression through mitosis until all of the sister kinetochore-microtubule pairs are bi-oriented on the metaphase plate (96). Bi-orientation is achieved when microtubules emanating from opposite spindle poles stably attach to sister chromatids and generate tension across their kinetochores. The SAC blocks the activity of $\mathrm{APC} / \mathrm{C}^{\mathrm{Cdc} 20}$, which targets cyclin $\mathrm{B} 1$ and securin for degradation after bi-orientation $(21,22,44,54,69,149)$. Cyclin B1 activates Cdk1, pushing the cell through the G2/M transition and regulating key events early in mitosis, including nuclear envelope breakdown (NEBD) and spindle assembly $(21,35,36,54,95)$. Additionally, both cyclin B1 and securin inhibit separase, a protease that cleaves the Scc1 subunit of centromeric cohesin rings that hold sister chromatids together prior to anaphase $(42,43,49,114,128,132$, 133). Securin is a stoichiometric inhibitor that blocks cohesin from accessing the active site of 
separase (149), whereas cyclin B1 inhibits separase activity via Cdk1-mediated phosphorylation (53). Thus, $\mathrm{APC} / \mathrm{C}^{\mathrm{Cdc20}}$-mediated degradation of cyclin B1 and securin promotes sister chromatid separation, whereas the SAC prevents the cell from entering anaphase by blocking APC/C ${ }^{\mathrm{Cdc} 20}$ activity until the checkpoint requirements are satisfied.

The MCC comprises BubR1, Mad2, Bub3, and Cdc20 (121). Although the MCC is a soluble inhibitor of APC/C $\mathrm{C}^{\mathrm{Cdc} 20}$, all of the SAC components first accumulate at unattached and/or lowtension kinetochores in an Aurora B- and Mps1-dependent fashion (17, 55, 56, 80, 88, 119, 123, $124,137)$. Of note, much of the pioneering work that determined the order and dependence of kinetochore localization was performed using the microtubule depolymerizing agent nocodazole and the microtubule stabilizer taxol. These agents have been and are still often used in parallel to compare and contrast cellular responses to unattached kinetochores (nocodazole) versus lowtension kinetochores (taxol). In this way, it was discovered that Mad2 primarily accumulates at unattached kinetochores, whereas Bub1-BubR1 accumulates in the context of low tension $(17,119$, 137). However, it is important to note that the distinction between unattached and low-tension kinetochores is not as simple as once thought. To that point, unattached kinetochores in the presence of nocodazole cannot generate tension. Comparatively, treatment with taxol hyperstabilizes microtubules, and hyperstabilized microtubules cannot generate tension across centromeres. In both cases, lack of tension activates the SAC leading to microtubule detachment (145). Nevertheless, these early experiments paved the way for our current understanding of kinetochore-mediated SAC signaling and MCC-mediated effector response.

Unattached and/or low-tension kinetochores emit a signal that ultimately mediates a SACdependent arrest until bi-orientation is established. Exactly how kinetochores emit this signal and mediate a response remains an active area of investigation. Nonetheless, our understanding of these processes has grown tremendously over the past two decades. Kinetochore-mediated SAC signaling is known to be highly dependent on two Mad-family proteins, Mad1 and Mad2. A key discovery in understanding SAC signaling was made when Mad1-Mad2 heterodimers were identified at unattached kinetochores (16). This critical discovery was supplemented by two landmark studies in which the kinetochore residence time for various SAC components was determined $(55,113)$. The most significant finding of these studies was that kinetochore-bound Mad2 was found to exist in two mutually exclusive pools: a highly stable pool with low turnover and a separate pool that cycles rapidly at the kinetochore. The interpretation of these results was greatly facilitated by studies showing that Mad2 existed in two distinct conformational states, an inactive open (o-Mad2) or active closed (c-Mad2) state $(24,85)$. Accordingly, the stable kinetochorebound Mad2 pool is that which associates with Mad1. Mad1 facilitates the conversion of o-Mad2 to c-Mad2 such that Mad1-c-Mad2 stably binds unattached kinetochores (86). The rapidly cycling Mad2 pool points to the critical, rate-limiting step in SAC signal amplification and MCC formation. In this step, kinetochore-bound Mad1-c-Mad2 transiently binds soluble o-Mad2 and catalyzes its conformational change from o-Mad2 to c-Mad2. This prion-like reaction amplifies the signal generated by unattached kinetochores and mediates the first step in effecting a SAC response because c-Mad2 is the sole Mad2 conformer able to bind Cdc20 (86). It is important to appreciate that $\mathrm{p} 31^{\text {comet }}$ is a competitive inhibitor of $\mathrm{c}-\mathrm{Mad} 2$ and thus acts as a negative regulator of the SAC $(142,144)$.

Although c-Mad2 has been reported to inhibit APC/C $\mathrm{C}^{\mathrm{Cdc} 20}$ by competing for the same $\mathrm{Cdc} 20$ binding site as the APC/C (58), it is more widely accepted that c-Mad2 and BubR1 combine to synergistically inhibit APC/C $\mathrm{Cdc2}^{\mathrm{C} 2}$ (28). The synergism between c-Mad2 and BubR1 in blocking APC/C $\mathrm{C}^{\mathrm{Cdc2} 2}$ activity appears to be both indirect, by preventing $\mathrm{Cdc} 20$ from interacting with the APC/C (Figure 2a), and direct, by inhibiting the enzymatic activity of APC/C that is already bound to Cdc20 (Figure $2 \boldsymbol{b}$ ). With respect to the indirect mechanism, c-Mad2-Cdc20 has 


\section{a Inhibitition of coactivation}

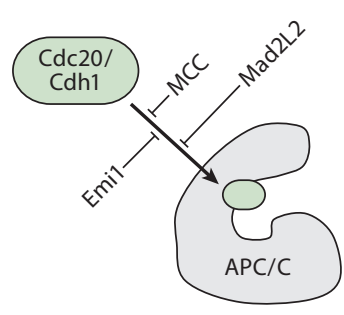

b

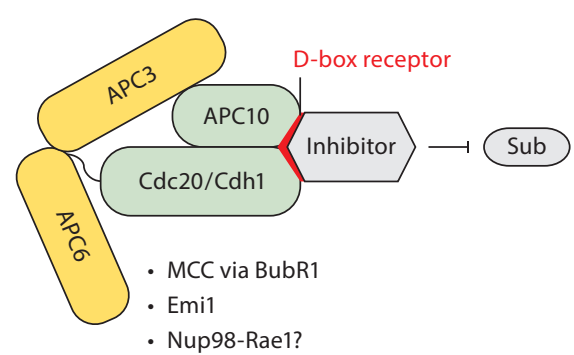

C Inhibition of ligase activity

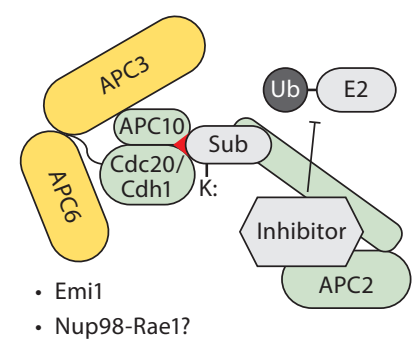

Figure 2

Molecular mechanisms of anaphase-promoting complex/cyclosome (APC/C) inhibition. (a) The mitotic checkpoint complex (MCC), early mitotic inhibitor 1 (Emi1), and Mad2L2 sequester Cdc20 or Cdh1 and prevent the coactivation of the APC/C. (b) Pseudosubstrate inhibitors, including BubR1 and Emil, bind the bipartite D-box receptor (shown in red) and prevent substrate (Sub) recruitment to the APC/C. (c) Emi1 inhibits ubiquitin (Ub) chain extension. The Nup98-Rae1 subcomplex is most likely to function as a ligase inhibitor; however, this hypothesis remains untested. The red triangle signifies the bipartite D-box receptor

been proposed to be an intermediate that induces a functional switch in Cdc20 to expose a previously inaccessible BubR1 binding domain (45). Accordingly, BubR1-Cdc20 complexes, but not c-Mad2-Cdc20 complexes, are sufficient to block the polyubiquitination of cyclin B1 in vitro (72). These results indicate that BubR1, but not c-Mad2, sequesters Cdc20 and prevents it from coactivating the APC/C. With respect to the direct mechanism of c-Mad2 and BubR1 synergy, c-Mad2 has been shown to position BubR1 optimally within the catalytic core of the APC/C to both disrupt the Cdc20-Apc10 bipartite D-box receptor and occupy the C-terminal WD40 $\beta$-propeller domain of Cdc20 to prevent $\operatorname{KEN-box-mediated~substrate~recruitment~}(13,51,75)$ (Figure $2 \boldsymbol{b}$ ). Remarkably, the indirect and direct mechanisms of c-Mad2 and BubR1-mediated $\mathrm{APC} / \mathrm{C}^{\mathrm{Cdc} 20}$ inhibition are not mutually exclusive. BubR1 has two N-terminal KEN-box degrons and a D-box just upstream of the second KEN-box motif (65). It was recently demonstrated that BubR1 can bind soluble Cdc20 through its first KEN-box and/or Cdc20 that is bound to APC/C through its D-box and second KEN-box (59). Taken together, unattached kinetochores elicit a SAC signal through the prion-like conversion of soluble o-Mad2 to c-Mad2, which binds Cdc20 to promote a more stable BubR1-Cdc20 complex not capable of interacting with the APC/C and/or ideally positions BubR1 to jam the catalytic core of the Cdc20-activated APC/C.

Historically, investigations into the nature of SAC signaling and the MCC were limited to the early stages of mitosis. However, a 2009 paper reported that BubR1 acts as a pseudosubstrate inhibitor of APC/C $\mathrm{C}^{\mathrm{Cdc} 20}$ during interphase in order to protect cyclin $\mathrm{B} 1$ from premature degradation (89). This was later followed up by the isolation of Mad2 and BubR1-containing interphase APC/C $\mathrm{C}^{\mathrm{Cdc} 20}$ complexes (88), demonstrating that $\mathrm{Cdc} 20$ coactivation of the APC/C takes place during interphase and requires MCC-mediated inhibition. However, because kinetochores do not exist until prometaphase, the platform from which the interphase APC/ $\mathrm{C}^{\mathrm{Cdc2} 2}$ inhibitor is generated remained enigmatic until recently. In 2014, it was discovered that nuclear pore complexes (NPCs) emit a wait-anaphase signal prior to mitosis (111). Intriguingly, Mad1-c-Mad2 heterodimers associate with the nuclear envelope (NE) during interphase because of Mad1's ability to bind the nuclear basket component Tpr. Interphase Mad1-c-Mad2 heterodimers catalyze the conformational change of soluble o-Mad2 to c-Mad2, which catalyzes the formation of interphase APC/C $\mathrm{C}^{\mathrm{Cdc2} 2}$ inhibitory complexes. Adding to the cross talk between NPCs and SAC components, Mps1, like Mad1 and Mad2, is bound to NPCs during interphase and is required 
to generate interphase APC/ $\mathrm{C}^{\mathrm{Cdc} 20}$ inhibitory complexes $(84,88,111)$. Thus, interphase NPCs and mitotic kinetochores play overlapping roles in mediating MCC formation, albeit in separate phases of the cell cycle.

Finally, how MCC-inhibited APC/C $\mathrm{C}^{\mathrm{Cdc} 20}$ is rapidly converted to active $\mathrm{APC} / \mathrm{C}^{\mathrm{Cdc} 20}$ upon biorientation remains a mystery. Nonetheless, this switch is known to be regulated by Ubch10-driven monoubiquitination and liberation of BubR1 and Mad2 from Cdc20 (107). The deubiquitinase Usp44 counteracts Ubch10-mediated ubiquitination of BubR1 and Mad2, and, accordingly, depletion of Usp44 abolishes SAC arrest even in the presence of normal checkpoint machinery (120). On the basis of these results, it is highly probable that there are multiple points of SAC and $\mathrm{APC} / \mathrm{C}^{\mathrm{Cdc} 20}$ regulation beyond the MCC that remain to be discovered.

Phosphoregulation. During prometaphase, the cell undergoes NEBD, allowing cyclin B1 to diffuse into the nucleoplasm and activate $\mathrm{Cdk} 1$. Cdk1 subsequently phosphorylates Cdh1, preventing Cdh1 from binding to the APC/C (146) (Figure 2a), thus ensuring that Cdc20 is the sole coactivator to associate with the APC/C. In metaphase, $\mathrm{APC} / \mathrm{C}^{\mathrm{Cdc} 20}$ polyubiquitinates cyclin $\mathrm{B} 1$ and steadily decreases its levels until anaphase. At the same time, Cdc14 phosphatase activity steadily increases. The combination of increased Cdc14-mediated Cdh1 dephosphorylation and decreased Cdk1 activity, along with the autoubiquitination of Cdc20 (30), triggers the interaction between $\mathrm{Cdh} 1$ and the APC/C following anaphase onset $(60,129)$. APC/C $\mathrm{C}^{\mathrm{Cdh} 1}$ remains active until the subsequent G1/S transition, at which point its activity might be quenched by a combination of three mechanisms. First, early mitotic inhibitor 1 (Emi1), an E2F target gene, binds and inactivates $\mathrm{APC} / \mathrm{C}^{\mathrm{Cdh} 1}(31,57,94,135)$. Second, following the destruction of Ubch10 in late G1 phase (106), cyclins A and E accumulate and reestablish the Cdk-dependent inhibitory phosphorylation of Cdh1 $(60,146)$. Finally, it has been reported that Cdh1 mediates its own autoubiquitination (81); however, this observation requires further investigation (104).

Early mitotic inhibitor 1. Emil is an inhibitor of interphase APC/C $\mathrm{C}^{\mathrm{Cdh} 1}$ complexes (108). In a similar fashion to the MCC, Emi1 has been reported to bind to the bipartite D-box coreceptor formed by Apc10 and Cdh1 through its C-terminal D-box motif (Figure $2 \boldsymbol{b}$ ) and to preferentially block ubiquitin chain extension $(31,94,135)$ (Figure 2 c). Following the G2/M transition, Emi1 is phosphorylated by Cdk1 and Polo-like kinase 1 (Plk1) and targeted for proteolysis by the SCF$\beta$-transducin repeat-containing protein ubiquitin ligase in late prophase $(48,90)$. The abrupt destruction of Emil was initially thought to activate the APC/C upon mitotic entry; however, subsequent studies revealed that Emi1-mediated inhibition of APC/C $\mathrm{C}^{\mathrm{Cdh} 1}$ is important to prevent DNA re-replication and polyploidization and does not affect the activity of the APC/C in mitosis $(25,87)$.

Mad2L2. Mad2L2 prevents Cdh1 from interacting with the APC/C through a direct proteinprotein interaction (82) (Figure 2a). Curiously, depletion of Mad2L2 results in the prometaphase destruction of select APC/C $\mathrm{C}^{\mathrm{Cdh} 1}$ substrates, including cyclin B1, securin, and Aurora A but not Ubch10. Thus, Mad2L2 ensures the robust transition from $\mathrm{APC} / \mathrm{C}^{\mathrm{Cdc} 20}$ to $\mathrm{APC} / \mathrm{C}^{\mathrm{Cdh} 1}$ activity during the metaphase-to-anaphase transition because Mad2L2 depletion results in premature association of APC/C with Cdh1 in prometaphase.

Nup98-Rae1 subcomplex. A subcomplex containing the soluble nuclear pore proteins Nup98 and Rae1 has also been shown to inhibit $\mathrm{APC} / \mathrm{C}^{\mathrm{Cdh} 1}$ activity $(62,63,100)$. Nup98-Rae1 binds and inhibits preformed $\mathrm{APC} / \mathrm{C}^{\mathrm{Cdh} 1}$ complexes in late $\mathrm{G} 2$ phase in order to prevent the premature 
degradation of securin and Plk1 (100). Although it has been shown using in vitro ubiquitination assays that Nup98-Rae1 prevents the APC/C ${ }^{\text {Cdh1 }}$-mediated polyubiquitination of securin (63), the precise mechanism of inhibition is unknown. Because Nup98-Rae1-APC/C Cdh1 complexes contain securin during mitosis (62), it is unlikely that Nup98-Rae1 behaves as a pseudosubstrate inhibitor akin to BubR1 (Figure $2 \boldsymbol{b}$ ). Rather, it is more likely that Nup98-Rae1 blocks ubiquitin chain initiation and/or extension (Figure 2c); however, this idea has never been tested.

\section{WHOLE-CHROMOSOMAL INSTABILITY: A FACILITATOR OF TUMORIGENESIS?}

To discuss the relationship between W-CIN, aneuploidy, and tumorigenesis, one must first appreciate the distinctions between aneuploidy and W-CIN. Aneuploidy describes the karyotype of a single somatic cell when it has anything other than two copies of each chromosome. Importantly, the term aneuploidy does not distinguish between whole-chromosome gain and loss. $\mathrm{W}$-CIN is the persistent inability of a cell to accurately segregate its genome in successive rounds of cell division, thus generating progeny, each with a different aneuploid karyotype (101, 103, 109). Thus, aneuploidy is an obligate outcome of W-CIN, but W-CIN is not the only cause of aneuploidy. For example, Down syndrome, which is characterized by trisomy of chromosome 21, usually arises from a spontaneous maternal nondisjunction event during meiosis rather than the persistent, random segregation of gamete chromosomes. Thus, patients with Down syndrome are said to exhibit stable aneuploidy rather than W-CIN (103).

Two types of CIN are widely recognized. The first type, W-CIN, results in the gain or loss of entire chromosomes following an aberrant mitosis. W-CIN is thought to arise from abnormal kinetochore-microtubule attachments. The second type of CIN, structural-CIN (S-CIN), can result in the gain or loss of chromosomal fragments, DNA breakage, and/or translocation events. $\mathrm{S}-\mathrm{CIN}$ is caused by chromatin bridging in anaphase and/or DNA replication/repair defects during interphase. Because of space constraints, mechanisms of S-CIN are not discussed. Please see the following reviews for more information on the causes and consequences of S-CIN: References 33 and 41 .

\section{Molecular Mechanisms of Aneuploidization}

To understand the precipitating events that lead to whole-chromosome gain or loss, it is necessary to first appreciate the importance of proper, amphitelic kinetochore-microtubule attachments during mitosis $(40,134)$. When microtubules emanating from opposite spindle poles attach exclusively to their proximal sister kinetochore, the attachment is said to be amphitelic (Figure 3). However, erroneous monotelic, syntelic, and merotelic attachments can also occur that, if left undetected and/or uncorrected, result in chromosome missegregation and aneuploidy (Figure 3). When only one of the two sister kinetochores of a chromosome is attached to microtubules originating from the proximal spindle pole, the attachment is said to be monotelic. In contrast, if both sister kinetochores are attached to microtubules emanating from the same spindle pole, the attachment is considered syntelic (Figure 3). Finally, if one sister kinetochore is properly attached to microtubules from its proximal spindle pole while the other kinetochore is attached to microtubules from both spindle poles, the attachment is called merotelic (Figure 3).

When determining the nature of the kinetochore-microtubule attachment defect(s) responsible for the generation of aneuploidy in a population of cells, one must determine the nature and frequency of chromosome missegregation events. Chromosome missegregation events are detected via time-lapse microscopy using living cells that express a fluorescent histone, whereas aneuploidy is determined by counting Giemsa-stained metaphase chromosomes, comparative genomic 


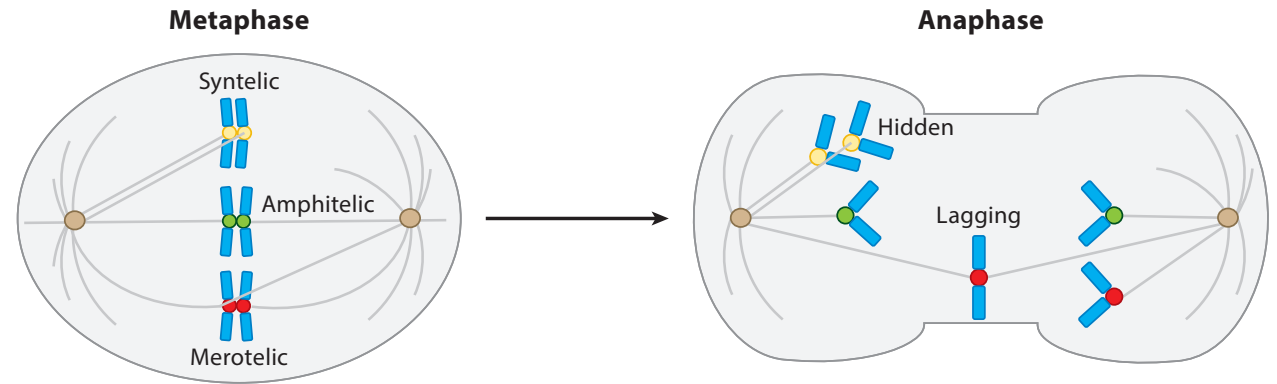

Figure 3

Cellular mechanisms of whole-chromosome aneuploidy. During metaphase, when microtubules emanating from each spindle pole attach to their proximal sister kinetochore, the attachment is said to be amphitelic (green). The result of this type of attachment is proper segregation of the sister chromatids into each daughter cell. When microtubules from one spindle pole attach to both sister kinetochores, a syntelic attachment is formed (yellow). Syntelic attachments cause chromosome missegregation in the form of hidden chromatids. Merotelic attachments (red) are formed when one sister kinetochore is properly attached to microtubules originating from its proximal spindle pole while the other kinetochore is attached to microtubules from both spindle poles. Merotelic attachments lead to lagging chromatids.

hybridization, fluorescence in situ hybridization, or spectral karyotyping. As is discussed below, it is critical to perform both karyotype and chromosome segregation analyses because certain defects are only detected by one method of experimentation.

Hidden chromatids. Hidden chromatids are aptly named because they are detectable by karyotype analysis but go unnoticed using chromosome segregation experimentation methods (127). Therefore, in a population of cells with widespread aneuploidy but without overt chromosome missegregation events, hidden chromosomes should be strongly suspected. Hidden chromatids are caused by syntelic attachments and generally align on the metaphase plate before segregating inappropriately to the wrong daughter cell (127) (Figure 3). Because the microtubule-generated pulling force is unopposed in this type of attachment error, the chromatid does not lag and segregates synchronously with the other sister chromatids. As a clarifying note, hidden refers to the behavior of the missegregating chromatid during anaphase. Hidden chromatids may initially misalign in metaphase, which can be readily detected using live-cell imaging techniques. The subsequent behavior of the misaligned chromatid pair following anaphase onset can help determine whether the defect arose from a syntelic or other attachment error. Accordingly, misalignments due to syntelic attachments lead to both sister chromatids segregating to the same spindle pole. Additionally, the misaligned chromatids progressively lose resolution and become obscured by the remainder of the chromatids as they synchronously move toward their respective spindle pole in anaphase, making them indistinguishable when using the time-lapse methods described. In contrast, syntelic misalignments that are corrected prior to anaphase onset result in chromatid lagging because the properly attached chromatid must travel a greater distance to reach its intended pole.

As discussed above, syntelic attachments activate the SAC because of a lack of interkinetochore tension. Improper kinetochore-microtubule attachments are destabilized by the kinase activity of Aurora B $(14,122)$. Kinetochore-microtubule destabilization results in a temporarily unattached kinetochore, which sustains SAC arrest until amphitelic attachments are established. Thus, in a population of cells with high incidence of hidden chromosome missegregation events, defective SAC signaling and/or defects in the Aurora B-driven error correction pathway should be considered. 
Lagging chromatids. In contrast to hidden chromatids, lagging chromatids can be detected by both karyotype analysis and live-cell analysis for chromosome segregation defects (Figure 3). Importantly, lagging chromatids result from merotelic attachments (18). Unlike monotelic and syntelic attachments, the SAC does not mediate arrest in the presence of merotelic attachments because merotelic attachments generate sufficient interkinetochore tension to satisfy the SAC $(19,40)$. The underlying cellular defects leading to lagging chromosomes can be broadly classified into two categories: (a) those that decrease the cell's ability to correct merotelic attachments and (b) those that increase the frequency of merotelic attachments.

Like syntelic attachments, merotelic attachments are corrected by the mitotic kinase Aurora B (74). Aurora B maintains chromosome segregation fidelity by phosphorylating components of the KMN (KNL1-Mis12-Ndc80) network and Ska (Ska1-3) complex in order to destabilize microtubule-kinetochore attachments in the absence of tension $(14,15,20,74,122)$. Under tension, inner centromeric Aurora B is physically separated from its outer kinetochore substrates and therefore cannot destabilize microtubule-kinetochore attachments $(20,83,139)$. Defects in any of the components of the error correction pathway, especially Aurora B, reduce merotelic error correction and promote lagging chromatid formation (20).

In addition to destabilizing the physical connections formed between kinetochores and microtubules by the phosphorylation of KMN network and Ska complex components, microtubules themselves must be sufficiently malleable to destabilization in order to facilitate error correction and ward off chromosome missegregation events $(7,37)$. Indeed, Aurora B has been shown to stimulate the microtubule-depolymerizing kinesins Kif2b and MCAK during prometaphase and metaphase, respectively (8). Furthermore, increased plus-end directed microtubule growth has also been demonstrated to promote merotely and W-CIN (27). The proposed mechanism for this includes transient spindle geometry defects, which have been suggested to reduce error correction through the inappropriate generation of tension between sister kinetochores (98); however, this idea has never been tested.

Because the SAC does not detect the presence of merotelic attachments, the rate at which a cell with merotelic attachments progresses through mitosis is limited by the activity of the Aurora Bdriven error correction machinery. Aurora B destabilizes kinetochore-microtubule attachments, thereby generating an unattached kinetochore and subsequently activating the SAC. However, if a cell proceeds too rapidly through mitosis, there may be insufficient time for the error correction machinery to destabilize erroneous attachments and activate the SAC. Thus, rapid mitotic progression predisposes cells to lagging chromatids even in the presence of a normal error correction pathway and functional SAC. This principle was elegantly demonstrated using a mutant form of $\operatorname{Mad} 1\left(\mathrm{Mad}^{\triangle \mathrm{NP} 2}\right)$ that could bind kinetochores and elicit a robust SAC response in the presence of nocodazole but could not bind NPCs, which is required to generate interphase APC/C $\mathrm{Cdc2}^{20}$ inhibitory complexes and prolong mitotic timing (111). Mad1 ${ }^{\triangle \mathrm{NP} 2}$ cells are prone to lagging chromatids, but, remarkably, this defect is restored when mitotic timing is extended using the APC/C inhibitor proTAME. This principle can likely be extrapolated to other mutations known to decrease mitotic timing, including knockout of Mps1 (88), Mad2 or Bub1 insufficiency (92, 102, 105, 143), or hemizygosity of a Cdc20 allele, Cdc20 AAA, that cannot bind Mad2 (79).

Alternatively, the known defects that enhance the formation of merotelic attachments are abnormal centrosome dynamics and centrosome amplification. Abnormal centrosome dynamics, which include centrosome disjunction and movement defects and lead to metaphase spindle asymmetry, have been extensively linked to the formation of merotelic attachments and lagging chromatids $(98-100,116,117,148)$. The principal idea is that delayed centrosome separation increases the frequency of syntelic and merotelic attachments because kinetochores in this scenario are equally accessible to microtubules emanating from both spindle poles $(116,117)$. It is 
unclear why the error correction machinery is unable to resolve all of the erroneous attachments generated by abnormal centrosome dynamics. Nonetheless, because the lack of a built-in merotely checkpoint predisposes cells in culture to a basal rate of lagging chromatids (125), the incidence of lagging chromatids is likely proportional with the number of merotelic attachments formed.

Finally, centrosome amplification is a major risk factor for the development of lagging chromatids and is perhaps the most relevant mechanism of W-CIN in cancer cells (131). Multipolar spindle intermediates encourage microtubules from two or more centrosomes to attach to a single kinetochore. However, the collapse of these multipolar intermediates into pseudobipolar spindles contorts microtubules in such a way that a single kinetochore can be connected to multiple centrosomes in either or both poles, thus establishing widespread merotely and promoting chromatid lagging (32). Centriole biogenesis, centrosome duplication, and their role in tumorigenesis have been reviewed elsewhere and are not covered in detail here $(38,52,98)$.

\section{THE RELATIONSHIP BETWEEN WHOLE-CHROMOSOMAL INSTABILITY, ANEUPLOIDY, AND CANCER}

Nearly every solid tumor and upward of half of all hematologic malignancies are aneuploid (112), and although there exists a strong correlation between aneuploidy and cancer, their precise relationship remains hotly debated. In the following section, we briefly consider how W-CIN might facilitate tumorigenesis in the context of tumor evolution, review the evidence demonstrating that aneuploidy can also suppress tumorigenesis, and provide a perspective that might help resolve some aspects of the apparent aneuploidy-cancer paradox.

\section{Whole-Chromosomal Instability as a Facilitator of Tumorigenesis}

In the early 1990s, the Vogelstein group proposed that colon cancer develops in a stepwise fashion and suggested that each step is associated with specific driver mutations (29, 76). According to this model, it can be inferred that W-CIN occurs during the early stages of colon cancer development. Specifically, inactivation of the adenomatous polyposis coli (APC) gene and persistent activation of the Ras signaling pathway, both of which are observed in early adenomas, have been shown to independently promote $\mathrm{W}$-CIN and aneuploidy in addition to sustaining cell growth and proliferation (101).

Since this model of colon carcinogenesis emerged, additional models of tumor evolution have been suggested. Importantly, W-CIN and the acquisition of an aneuploid karyotype remain early features in nearly every model. For example, whole-genome and exome single-cell sequencing from estrogen-receptor-positive and triple-negative breast cancer specimens revealed that aneuploidization occurs in a punctuated burst in the initiating stages of tumor development (136). Interestingly, the aneuploid karyotypes were faithfully propagated thereafter, whereas the frequency of point mutations increased over time. This suggests that $\mathrm{W}-\mathrm{CIN}$ is a critical barrier that must be overcome in the initial stages of breast cancer development, whereas genomic instability aids in its maintenance and progression.

The idea that the early acquisition of W-CIN facilitates tumorigenesis is supported by the observation that many $\mathrm{W}$-CIN mouse models are tumor prone and/or accelerate tumor onset when crossed onto sensitized genetic backgrounds (110). One of the most likely mechanistic explanations for why W-CIN might facilitate tumor initiation is that aberrant mitoses may facilitate loss of critical tumor suppressor genes. Indeed, this was shown to be the case in Bub1-insufficient mice that also lacked one copy of the $p 53$ gene or had a mutated $A P C$ allele (4). 


\section{Whole-Chromosomal Instability as a Tumor Suppressor}

Considering the high correlation between aneuploidy and cancer, the nearly universal consensus that aneuploidization occurs early in most models of tumor evolution, the experimental evidence implicating W-CIN in loss of tumor suppressor heterozygosity, and the observation that many aneuploidy-prone mouse models display increased tumor susceptibility, it would seem logical to conclude that W-CIN facilitates tumorigenesis. However, there is also a large body of evidence suggesting that $\mathrm{W}$-CIN and aneuploidy can have no impact on or even suppress tumorigenesis, calling into question the idea that $\mathrm{W}$-CIN is a universal facilitator of tumorigenesis. As this topic has been covered extensively elsewhere $(39,103)$, we briefly summarize the main counterarguments to $\mathrm{W}$-CIN as a facilitator of tumorigenesis before offering a few perspectives for resolving the apparent paradox.

First, although many mouse models of $\mathrm{W}$-CIN-induced aneuploidy are tumor prone, several are not (110). The most robust example of this is the $B u b 3^{+/-}$Rae $1^{+/-}$mouse model, which, despite having similar in vivo rates of aneuploidy as other tumor-prone W-CIN models, shows no predisposition to spontaneous tumorigenesis (3). Accordingly, if W-CIN was a universal facilitator of tumorigenesis, one would expect $B u b 3^{+/-} \mathrm{Rae} 1^{+/-}$mice to display increased tumor susceptibility. Moreover, not only has W-CIN been shown to be tumor neutral, as in the case of $B u b 3^{+/-} R a e 1^{+-}$ mice, but aneuploidy has also been demonstrated to be overtly tumor suppressive. In a landmark study, it was demonstrated that trisomy cell lines proliferate slower and show delayed spontaneous immortalization compared with euploid control lines (140). Together, these studies highlight the variation in aneuploidy-induced phenotypes, spanning the full spectrum of tumor promoting to tumor neutral to tumor suppressing, calling into question the universality of W-CIN as a tumor facilitator.

Second, although W-CIN promotes loss of tumor suppressor heterozygosity, W-CIN and aneuploidy have also been shown to elicit a large spectrum of counteractive tumor-suppressive stress responses, including cell cycle arrest $(126,140)$, programmed cell death $(61,70,78,115)$, mitotic catastrophe (130), and senescence (77). Thus, it is difficult to predict whether W-CIN will tip the balance in favor of tumor promotion via loss of critical tumor suppressor genes or tumor suppression by upregulating a cellular stress response pathway, again arguing against the universality of W-CIN as a tumor facilitator.

\section{Perspectives on Reconciling the Aneuploidy-Cancer Paradox}

Models that attempt to integrate the totality of evidence regarding the relationship between aneuploidy and cancer into a cohesive understanding of how W-CIN impacts tumor development have failed to gain widespread acceptance. One explanation for the differing effects of W-CIN on tumor development is the gene-centric theory, which states that the specific aneuploidy-inducing gene, its W-CIN-independent cellular functions, the nature of the genetic defect, the cell type and organ system affected, and/or the ability to cooperate or synergize with other cancer-causing mutations explain how aneuploidy effects tumor development in that particular genetic context $(109,110)$. This is a helpful framework to explain why $B u b 3^{+/-}$mice are not susceptible to spontaneous tumorigenesis, whereas $\operatorname{RanBP} 2^{\mathrm{H} / \mathrm{H}}$ and $\operatorname{RanBP} 2^{-/ \mathrm{H}}$ mice are prone to forming spontaneous tumors despite having similar rates of in vivo aneuploidy (110). However, the main limitation of the gene-centric theory is that it places too much emphasis on the W-CIN-independent cellular functions of the affected gene, thus relegating the relative contribution of $\mathrm{W}$-CIN-induced aneuploidy to the tumor phenotype. Moreover, seeing as mutations in putative mitosis genes are rare in human cancers (101), the gene-centric theory seems insufficient to explain how W-CIN 
contributes to the development of human cancers that do not harbor mutations in Bub1, Bub1b, Mad1, Mad2, CenpE, etc.

The second explanation for the differing effect of W-CIN on tumor development is the rate theory. The rate theory states that the rate of chromosome missegregation events either promotes or suppresses tumorigenesis $(115,138)$. The basis for this theory comes from studies using $\operatorname{Cenp}^{+/-}$mice. It was noted that these mice were prone to forming lung adenomas and splenic lymphomas yet were protected against hepatocellular carcinomas (138). It was postulated that this paradoxical finding was dependent on the rate of chromosome missegregation events in the context of CenpE heterozygosity, with moderate rates of missegregation events predisposing to transformation and tumorigenesis, whereas high rates evoked cell death. Accordingly, the combination of basal chromosome missegregation events in the liver plus CenpE heterozygosity-induced missegregation evoked such a high rate of aneuploidy that the scales were tipped in favor of cell death rather than transformation $(115,138)$. Importantly, this model has been validated using gene expression profiles of various human cancers, demonstrating that patients with tumors with the highest levels of W-CIN had a better prognosis than those with intermediate levels of W-CIN (9). On the basis of this model, a plausible anticancer strategy would be to increase chromosome missegregation rates-perhaps by targeting one of the master mitotic kinases such as Plk1, Cdk1/2, or Aurora $\mathrm{A} / \mathrm{B}$ - in order to elicit a cell death response. Such a strategy would parallel the rationale behind the development of Chk1/2 and Wee1/2 inhibitors.

A third explanation for why aneuploidy might both facilitate and inhibit tumorigenesis is the gain/loss theory (Figure 4). This model recognizes the term aneuploidy as an umbrella, catchall term that includes any somatic cell with a nondiploid karyotype. Accordingly, cells with net chromosome loss and those with net chromosome gain are likely to have divergent cellular responses even though both cells are considered aneuploid. The gain/loss theory simultaneously affirms the widely accepted hypothesis that W-CIN facilitates the early stages of tumorigenesis through loss of tumor suppressor heterozygosity while recognizing the tumor-suppressive effects of whole-chromosome gain via proteotoxicity, cell cycle arrest, programmed cell death, mitotic catastrophe, and senescence. That is, the gain/loss theory views the apparent aneuploidy-cancer paradox as being different sides of the same coin. After all, following a missegregation event, both daughters are aneuploid, albeit one has lost chromosomal content and the other has gained chromosomal content. The independent cellular responses following such an event need not be linked in any way. This theory is partially supported by the finding that cancer cells tend to lose rather than gain chromosomes (26).

In general, it is the authors' view that W-CIN is a key facilitator of tumorigenesis. This is largely concluded on the basis of two key pieces of circumstantial evidence: $(a)$ The overwhelming majority of tumors are aneuploid, and $(b)$ a shared consequence of oncogene activation or tumor suppressor inactivation is W-CIN (101). Thus, it seems as though the most commonly affected cancer-critical genes are multifunctional and play a role in maintaining chromosomal stability in addition to modulating or mediating key signaling pathways, although this hypothesis requires meticulous experimentation.

Although we maintain the perspective that aneuploidy is a key facilitator of tumorigenesis, we reject the notion that the sole outcome of W-CIN is cancer. Nonetheless, this does not preclude one from concluding that Boveri was correct in hypothesizing that aneuploidy is a driving force of tumorigenesis. By way of analogy, it is colloquially recognized that smoking cigarettes causes lung cancer. This is based on the observation that smokers are up to 25 times more likely to develop lung cancer than nonsmokers. However, not every person with a significant history of cigarette smoking develops lung cancer. In fact, only a very small percentage of current or former smokers develop lung cancer. Instead, many smokers either succumb to chronic lung disease, heart 


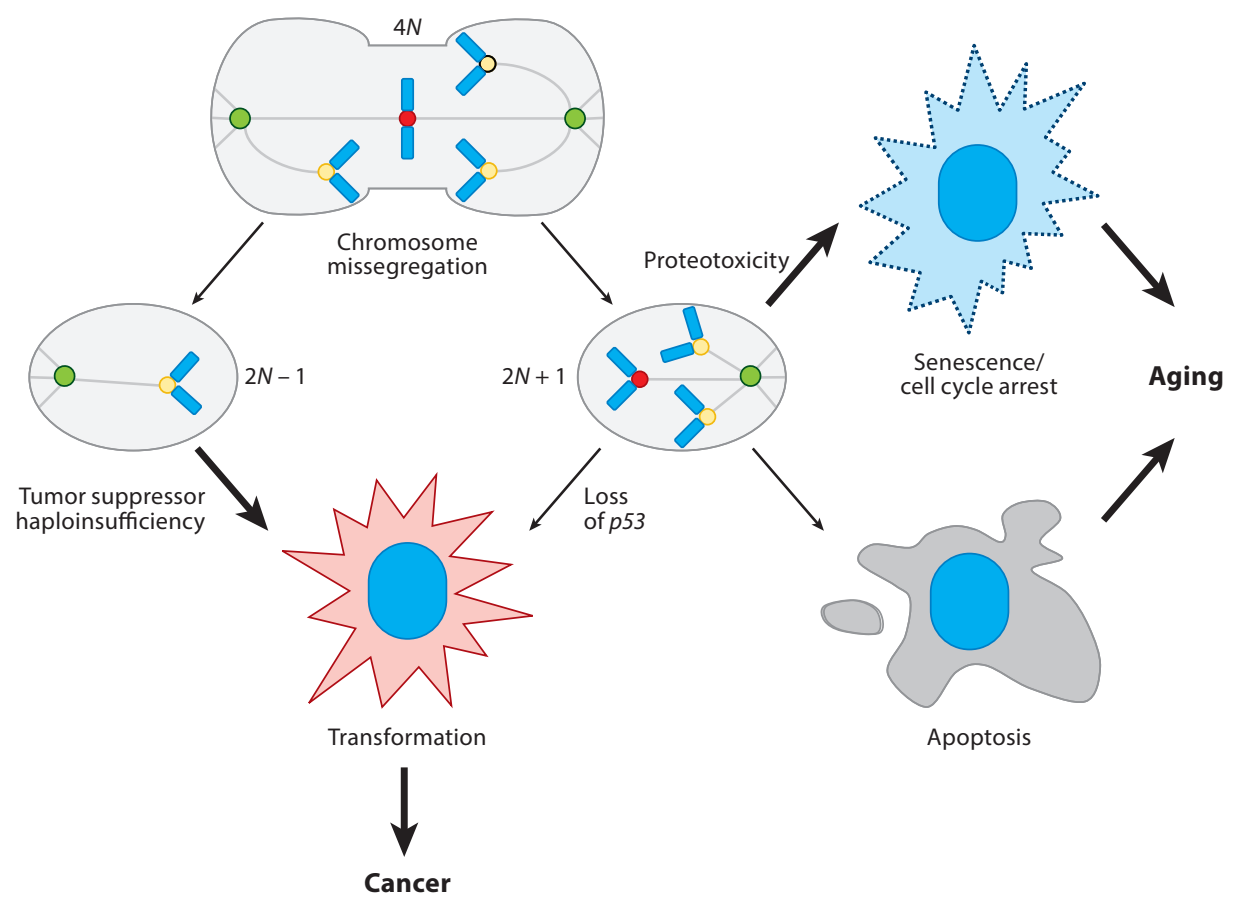

Figure 4

The gain/loss theory. Following a gross chromosome missegregation event, two aneuploid daughter cells exist, one with a net chromosome loss $(2 N-1)$ and the other with a net chromosome gain $(2 N+1)$. One fate of the $2 N-1$ cell is to undergo transformation because of the loss of tumor suppressors. The $2 N+1$ cell is more apt to undergo apoptosis or senescence/cell cycle arrest owing to increased proteotoxicity, which together promote aging.

disease, vascular disease, or, miraculously, remain in good health until late life. Yet, smoking is still considered the chief risk factor for lung cancer. On the basis of this parallelism, it is not illogical to conclude that $\mathrm{W}$-CIN is a facilitator or risk factor for developing cancer. Just as the vast majority of lung cancer cases occur in current or former smokers, the vast majority of tumors display prominent W-CIN and aneuploidy. Likewise, only a subset of smokers develop lung cancer, just as only a subset of aneuploid-prone mouse models are predisposed to cancer. And finally, just as smokers are predisposed to a gamut of devastating health outcomes and display significant clinical heterogeneity, so too does aneuploidy result in divergent cell fates leading to considerable physiologic heterogeneity.

\section{UNCOVERING AN UNEXPECTED LINK BETWEEN ANEUPLOIDY AND AGING}

With the objective to critically test Boveri's hypothesis, a cohort of mice with low levels of BubR1 $\left(B u b 1 b^{\mathrm{H} / \mathrm{H}}\right)$ and rapidly progressive aneuploidy were generated and evaluated for their susceptibility to form spontaneous and carcinogen-induced tumors $(2,3)$. Unexpectedly, Bub1b $b^{\mathrm{H} / \mathrm{H}}$ mice displayed several prominent progeroid phenotypes, including cataracts, kyphosis, impaired wound healing, growth retardation, and subdermal fat loss, by just three months of age. Additionally, Bub1b $b^{\mathrm{H} / \mathrm{H}}$ mice had a markedly reduced life span, with a median survival of six months. 
Notably, these mice were later found to be highly sensitive to forming carcinogen-induced tumors but did not live long enough to properly assess their susceptibility to spontaneous tumorigenesis (3). Less than two years after the initial description of the BubR1 hypomorphic mouse model, a second aneuploidy-prone mouse model double haploinsufficient for Bub3 and Rae1 was also shown to display similar progeroid features, albeit at a later time point than the $B u b 1 b^{\mathrm{H} / \mathrm{H}}$ model (3).

Together, these initial studies suggested a causal relationship between W-CIN and aging. This link was further strengthened in 2013 when it was discovered that BubR1 overexpression in otherwise wild-type mice prevented age-related aneuploidization, protected against tumorigenesis, delayed multiple aging phenotypes, and extended life span (1). The aneuploidy-aging theory also gained significant traction with the clinical description of mosaic-variegated aneuploidy, an extremely rare human syndrome characterized by widespread karyotypic mosaicism, early cataract and cancer onset, facial dysmorphism, growth retardation, and shortened life span (47).

Despite these findings, there remains a lack of indisputable evidence demonstrating that aneuploidy per se is a driver or facilitator of aging. In particular, the premature aging phenotype of BubR1 hypomorphic mice was subsequently shown to be senescence dependent, driven by the upregulation of $\mathrm{p} 16^{\mathrm{Ink} 4 \mathrm{a}}(5,6)$. Whether progressive aneuploidy is the stimulus for p16-mediated senescence in $B u b 1 b^{\mathrm{H} / \mathrm{H}}$ mice has not been directly investigated, although this is an attractive hypothesis for why W-CIN may facilitate both cancer and aging (Figure 4). Furthermore, whether systemic, in vivo aneuploidy is attenuated upon the removal of senescent cells or in the genetic context of $p 16^{-/-} \mathrm{Bub} 1 b^{\mathrm{H} / \mathrm{H}}$ is also unknown. Additionally, there are several mouse models of $\mathrm{W}$-CIN in which accelerated aging either did not occur, as in the case of Bub1 hypomorphs (61), or was never investigated, most likely because of lack of conspicuous features of accelerated aging. Thus, although circumstantial evidence has linked W-CIN and aging, there remains a great need to more systematically and directly test this hypothesis.

\section{CONCLUDING REMARKS AND FUTURE DIRECTIONS}

Great advances have been made in understanding the molecular and biochemical mechanisms underlying mitosis. Furthermore, current paradigms suggest that the inability to accurately and reproducibly segregate genomic information in successive rounds of cell division facilitate malignant transformation and aging. The challenge that lies ahead-the next great frontier for the field-is to translate this knowledge into clinically relevant solutions for patients. We can be encouraged that some of the most widespread and successful anti-cancer therapeutics are those that directly target mitosis, namely the vinca alkaloids and taxanes. Yet the search for more-targeted, less-toxic alternatives remains. CIN in rapidly dividing cancer cells may open a therapeutic window with which novel agents might exacerbate aneuploidy to arouse the cell's intrinsic anti-cancer mechanisms (e.g., apoptosis, senescence) while leaving nonmalignant cells unharmed. Aneuploidy may alternatively reconfigure a cell's metabolism, increasing its susceptibility to specific classes of pharmacological agents. Whatever the case, our challenge is to take the information that has been so diligently and laboriously obtained over the past 25 years and turn it into something that improves the quality and quantity of patients' lives.

\section{DISCLOSURE STATEMENT}

The authors are not aware of any affiliations, memberships, funding, or financial holdings that might be perceived as affecting the objectivity of this review. 


\section{ACKNOWLEDGMENTS}

We thank Darren Baker, Khaled Aziz, and Cynthia Sieben for critical reading of the manuscript. This work was supported by grants from the NIH (F30 CA189339 to R.M. Naylor and R01 CA096985, R01 CA168709, and R01 CA126828 to J.M. van Deursen).

\section{LITERATURE CITED}

1. Baker DJ, Dawlaty MM, Wijshake T, Jeganathan KB, Malureanu L, et al. 2013. Increased expression of BubR1 protects against aneuploidy and cancer and extends healthy lifespan. Nat. Cell Biol. 15:96-102

2. Baker DJ, Jeganathan KB, Cameron JD, Thompson M, Juneja S, et al. 2004. BubR1 insufficiency causes early onset of aging-associated phenotypes and infertility in mice. Nat. Genet. 36:744-49

3. Baker DJ, Jeganathan KB, Malureanu L, Perez-Terzic C, Terzic A, van Deursen JM. 2006. Early agingassociated phenotypes in Bub3/Rae1 haploinsufficient mice. 7. Cell Biol. 172:529-40

4. Baker DJ, Jin F, Jeganathan KB, van Deursen JM. 2009. Whole chromosome instability caused by Bub1 insufficiency drives tumorigenesis through tumor suppressor gene loss of heterozygosity. Cancer Cell $16: 475-86$

5. Baker DJ, Perez-Terzic C, Jin F, Pitel K, Niederlander NJ, et al. 2008. Opposing roles for p16Ink4a and p19Arf in senescence and ageing caused by BubR1 insufficiency. Nat. Cell Biol. 10:825-36

6. Baker DJ, Wijshake T, Tchkonia T, LeBrasseur NK, Childs BG, et al. 2011. Clearance of p16Ink4apositive senescent cells delays ageing-associated disorders. Nature 479:232-36

7. Bakhoum SF, Genovese G, Compton DA. 2009. Deviant kinetochore microtubule dynamics underlie chromosomal instability. Curr. Biol. 19:1937-42

8. Bakhoum SF, Thompson SL, Manning AL, Compton DA. 2009. Genome stability is ensured by temporal control of kinetochore-microtubule dynamics. Nat. Cell Biol. 11:27-35

9. Birkbak NJ, Eklund AC, Li Q, McClelland SE, Endesfelder D, et al. 2011. Paradoxical relationship between chromosomal instability and survival outcome in cancer. Cancer Res. 71:3447-52

10. Boveri T. 2008. Concerning the origin of malignant tumours by Theodor Boveri. Translated and annotated by Henry Harris. 7. Cell Sci. 121(Suppl. 1):1-84

11. Brown NG, Watson ER, Weissmann F, Jarvis MA, VanderLinden R, et al. 2014. Mechanism of polyubiquitination by human anaphase-promoting complex: RING repurposing for ubiquitin chain assembly. Mol. Cell 56:246-60

12. Chang L, Zhang Z, Yang J, McLaughlin SH, Barford D. 2014. Molecular architecture and mechanism of the anaphase-promoting complex. Nature 513:388-93

13. Chao WC, Kulkarni K, Zhang Z, Kong EH, Barford D. 2012. Structure of the mitotic checkpoint complex. Nature 484:208-13

14. Cheeseman IM, Anderson S, Jwa M, Green EM, Kang J, et al. 2002. Phospho-regulation of kinetochoremicrotubule attachments by the Aurora kinase Ipl1p. Cell 111:163-72

15. Cheeseman IM, Chappie JS, Wilson-Kubalek EM, Desai A. 2006. The conserved KMN network constitutes the core microtubule-binding site of the kinetochore. Cell 127:983-97

16. Chen RH, Shevchenko A, Mann M, Murray AW. 1998. Spindle checkpoint protein Xmad1 recruits Xmad2 to unattached kinetochores. F. Cell Biol. 143:283-95

17. Chen RH, Waters JC, Salmon ED, Murray AW. 1996. Association of spindle assembly checkpoint component XMAD2 with unattached kinetochores. Science 274:242-46

18. Cimini D, Cameron LA, Salmon ED. 2004. Anaphase spindle mechanics prevent mis-segregation of merotelically oriented chromosomes. Curr. Biol. 14:2149-55

19. Cimini D, Howell B, Maddox P, Khodjakov A, Degrassi F, Salmon ED. 2001. Merotelic kinetochore orientation is a major mechanism of aneuploidy in mitotic mammalian tissue cells. F. Cell Biol. 153:517-27

20. Cimini D, Wan X, Hirel CB, Salmon ED. 2006. Aurora kinase promotes turnover of kinetochore microtubules to reduce chromosome segregation errors. Curr. Biol. 16:1711-18

21. Clute P, Pines J. 1999. Temporal and spatial control of cyclin B1 destruction in metaphase. Nat. Cell Biol. 1:82-87 
22. Cohen-Fix O, Peters JM, Kirschner MW, Koshland D. 1996. Anaphase initiation in Saccharomyces cerevisiae is controlled by the APC-dependent degradation of the anaphase inhibitor Pds1p. Genes Dev. 10:3081-93

23. da Fonseca PC, Kong EH, Zhang Z, Schreiber A, Williams MA, et al. 2011. Structures of APC/C(Cdh1) with substrates identify Cdh1 and Apc10 as the D-box co-receptor. Nature 470:274-78

24. De Antoni A, Pearson CG, Cimini D, Canman JC, Sala V, et al. 2005. The Mad1/Mad2 complex as a template for Mad2 activation in the spindle assembly checkpoint. Curr. Biol. 15:214-25

25. Di Fiore B, Pines J. 2007. Emil is needed to couple DNA replication with mitosis but does not regulate activation of the mitotic APC/C. 7. Cell Biol. 177:425-37

26. Duijf PH, Schultz N, Benezra R. 2013. Cancer cells preferentially lose small chromosomes. Int. F. Cancer. 132:2316-26

27. Ertych N, Stolz A, Stenzinger A, Weichert W, Kaulfuss S, et al. 2014. Increased microtubule assembly rates influence chromosomal instability in colorectal cancer cells. Nat. Cell Biol. 16:779-91

28. Fang G. 2002. Checkpoint protein BubR1 acts synergistically with Mad2 to inhibit anaphase-promoting complex. Mol. Biol. Cell 13:755-66

29. Fearon ER, Vogelstein B. 1990. A genetic model for colorectal tumorigenesis. Cell 61:759-67

30. Foster SA, Morgan DO. 2012. The APC/C subunit Mnd2/Apc15 promotes Cdc20 autoubiquitination and spindle assembly checkpoint inactivation. Mol. Cell 47:921-32

31. Frye JJ, Brown NG, Petzold G, Watson ER, Grace CR, et al. 2013. Electron microscopy structure of human APC/C(CDH1)-EMI1 reveals multimodal mechanism of E3 ligase shutdown. Nat. Struct. Mol. Biol. 20:827-35

32. Ganem NJ, Godinho SA, Pellman D. 2009. A mechanism linking extra centrosomes to chromosomal instability. Nature 460:278-82

33. Ganem NJ, Pellman D. 2012. Linking abnormal mitosis to the acquisition of DNA damage. 7. Cell Biol. 199:871-81

34. Garnett MJ, Mansfeld J, Godwin C, Matsusaka T, Wu J, et al. 2009. UBE2S elongates ubiquitin chains on APC/C substrates to promote mitotic exit. Nat. Cell Biol. 11:1363-69

35. Gautier J, Minshull J, Lohka M, Glotzer M, Hunt T, Maller JL. 1990. Cyclin is a component of maturation-promoting factor from Xenopus. Cell 60:487-94

36. Gavet O, Pines J. 2010. Progressive activation of cyclinB1-Cdk1 coordinates entry to mitosis. Dev. Cell 18:533-43

37. Godek KM, Kabeche L, Compton DA. 2015. Regulation of kinetochore-microtubule attachments through homeostatic control during mitosis. Nat. Rev. Mol. Cell Biol. 16:57-64

38. Gonczy P. 2012. Towards a molecular architecture of centriole assembly. Nat. Rev. Mol. Cell Biol. 13:42535

39. Gordon DJ, Resio B, Pellman D. 2012. Causes and consequences of aneuploidy in cancer. Nat. Rev. Genet. 13:189-203

40. Gregan J, Polakova S, Zhang L, Tolic-Norrelykke IM, Cimini D. 2011. Merotelic kinetochore attachment: causes and effects. Trends Cell Biol. 21:374-81

41. Haarhuis JH, Elbatsh AM, Rowland BD. 2014. Cohesin and its regulation: on the logic of X-shaped chromosomes. Dev. Cell 31:7-18

42. Haering CH, Farcas AM, Arumugam P, Metson J, Nasmyth K. 2008. The cohesin ring concatenates sister DNA molecules. Nature 454:297-301

43. Haering CH, Lowe J, Hochwagen A, Nasmyth K. 2002. Molecular architecture of SMC proteins and the yeast cohesin complex. Mol. Cell 9:773-88

44. Hagting A, Den Elzen N, Vodermaier HC, Waizenegger IC, Peters JM, Pines J. 2002. Human securin proteolysis is controlled by the spindle checkpoint and reveals when the APC/C switches from activation by Cdc20 to Cdh1. 7. Cell Biol. 157:1125-37

45. Han JS, Holland AJ, Fachinetti D, Kulukian A, Cetin B, Cleveland DW. 2013. Catalytic assembly of the mitotic checkpoint inhibitor BubR1-Cdc20 by a Mad2-induced functional switch in Cdc20. Mol. Cell 51:92-104

46. Hanahan D, Weinberg RA. 2011. Hallmarks of cancer: the next generation. Cell 144:646-74 
47. Hanks S, Coleman K, Reid S, Plaja A, Firth H, et al. 2004. Constitutional aneuploidy and cancer predisposition caused by biallelic mutations in BUB1B. Nat. Genet. 36:1159-61

48. Hansen DV, Loktev AV, Ban KH, Jackson PK. 2004. Plk1 regulates activation of the anaphase promoting complex by phosphorylating and triggering SCF $\beta$ TrCP-dependent destruction of the APC inhibitor Emi1. Mol. Biol. Cell 15:5623-34

49. Hauf S, Waizenegger IC, Peters JM. 2001. Cohesin cleavage by separase required for anaphase and cytokinesis in human cells. Science 293:1320-23

50. He J, Chao WC, Zhang Z, Yang J, Cronin N, Barford D. 2013. Insights into degron recognition by APC/C coactivators from the structure of an Acm1-Cdh1 complex. Mol. Cell 50:649-60

51. Herzog F, Primorac I, Dube P, Lenart P, Sander B, et al. 2009. Structure of the anaphase-promoting complex/cyclosome interacting with a mitotic checkpoint complex. Science 323:1477-81

52. Holland AJ, Lan W, Cleveland DW. 2010. Centriole duplication: a lesson in self-control. Cell Cycle 9:2731-36

53. Holland AJ, Taylor SS. 2006. Cyclin-B1-mediated inhibition of excess separase is required for timely chromosome disjunction. 7. Cell Sci. 119:3325-36

54. Holloway SL, Glotzer M, King RW, Murray AW. 1993. Anaphase is initiated by proteolysis rather than by the inactivation of maturation-promoting factor. Cell 73:1393-402

55. Howell BJ, Hoffman DB, Fang G, Murray AW, Salmon ED. 2000. Visualization of Mad2 dynamics at kinetochores, along spindle fibers, and at spindle poles in living cells. F. Cell Biol. 150:1233-50

56. Howell BJ, Moree B, Farrar EM, Stewart S, Fang G, Salmon ED. 2004. Spindle checkpoint protein dynamics at kinetochores in living cells. Curr. Biol. 14:953-64

57. Hsu JY, Reimann JD, Sorensen CS, Lukas J, Jackson PK. 2002. E2F-dependent accumulation of hEmi1 regulates $S$ phase entry by inhibiting APC(Cdh1). Nat. Cell Biol. 4:358-66

58. Izawa D, Pines J. 2012. Mad2 and the APC/C compete for the same site on Cdc20 to ensure proper chromosome segregation. F. Cell Biol. 199:27-37

59. Izawa D, Pines J. 2015. The mitotic checkpoint complex binds a second CDC20 to inhibit active APC/C. Nature 517:631-34

60. Jaspersen SL, Charles JF, Morgan DO. 1999. Inhibitory phosphorylation of the APC regulator Hct1 is controlled by the kinase Cdc28 and the phosphatase Cdc14. Curr. Biol. 9:227-36

61. Jeganathan K, Malureanu L, Baker DJ, Abraham SC, van Deursen JM. 2007. Bub1 mediates cell death in response to chromosome missegregation and acts to suppress spontaneous tumorigenesis. 7 . Cell Biol. 179:255-67

62. Jeganathan KB, Baker DJ, van Deursen JM. 2006. Securin associates with APCCdh1 in prometaphase but its destruction is delayed by Rae1 and Nup98 until the metaphase/anaphase transition. Cell Cycle $5: 366-70$

63. Jeganathan KB, Malureanu L, van Deursen JM. 2005. The Rae1-Nup98 complex prevents aneuploidy by inhibiting securin degradation. Nature 438:1036-39

64. Jin L, Williamson A, Banerjee S, Philipp I, Rape M. 2008. Mechanism of ubiquitin-chain formation by the human anaphase-promoting complex. Cell 133:653-65

65. Kapanidou M, Lee S, Bolanos-Garcia VM. 2015. BubR1 kinase: protection against aneuploidy and premature aging. Trends Mol. Med. 21:364-72

66. Kelly A, Wickliffe KE, Song L, Fedrigo I, Rape M. 2014. Ubiquitin chain elongation requires E3dependent tracking of the emerging conjugate. Mol. Cell 56:232-45

67. Kerscher O, Felberbaum R, Hochstrasser M. 2006. Modification of proteins by ubiquitin and ubiquitinlike proteins. Annu. Rev. Cell Dev. Biol. 22:159-80

68. Kimata Y, Baxter JE, Fry AM, Yamano H. 2008. A role for the Fizzy/Cdc20 family of proteins in activation of the APC/C distinct from substrate recruitment. Mol. Cell 32:576-83

69. King RW, Peters JM, Tugendreich S, Rolfe M, Hieter P, Kirschner MW. 1995. A 20S complex containing CDC27 and CDC16 catalyzes the mitosis-specific conjugation of ubiquitin to cyclin B. Cell 81:279-88

70. Kops GJ, Foltz DR, Cleveland DW. 2004. Lethality to human cancer cells through massive chromosome loss by inhibition of the mitotic checkpoint. PNAS 101:8699-704 
71. Kraft C, Vodermaier HC, Maurer-Stroh S, Eisenhaber F, Peters JM. 2005. The WD40 propeller domain of Cdh1 functions as a destruction box receptor for APC/C substrates. Mol. Cell 18:543-53

72. Kulukian A, Han JS, Cleveland DW. 2009. Unattached kinetochores catalyze production of an anaphase inhibitor that requires a Mad2 template to prime Cdc20 for BubR1 binding. Dev. Cell 16:105-17

73. Labit H, Fujimitsu K, Bayin NS, Takaki T, Gannon J, Yamano H. 2012. Dephosphorylation of Cdc20 is required for its C-box-dependent activation of the APC/C. EMBO 7. 31:3351-62

74. Lampson MA, Cheeseman IM. 2011. Sensing centromere tension: Aurora B and the regulation of kinetochore function. Trends Cell Biol. 21:133-40

75. Lara-Gonzalez P, Scott MI, Diez M, Sen O, Taylor SS. 2011. BubR1 blocks substrate recruitment to the APC/C in a KEN-box-dependent manner. F. Cell Sci. 124:4332-45

76. Lengauer C, Kinzler KW, Vogelstein B. 1997. Genetic instability in colorectal cancers. Nature 386:62327

77. Lentini L, Barra V, Schillaci T, Di Leonardo A. 2012. MAD2 depletion triggers premature cellular senescence in human primary fibroblasts by activating a 53 pathway preventing aneuploid cells propagation. 7. Cell. Physiol. 227:3324-32

78. Li M, Fang X, Baker DJ, Guo L, Gao X, et al. 2010. The ATM-p53 pathway suppresses aneuploidyinduced tumorigenesis. PNAS 107:14188-93

79. Li M, Fang X, Wei Z, York JP, Zhang P. 2009. Loss of spindle assembly checkpoint-mediated inhibition of Cdc20 promotes tumorigenesis in mice. f. Cell Biol. 185:983-94

80. Li Y, Benezra R. 1996. Identification of a human mitotic checkpoint gene: bsMAD2. Science 274:246-48

81. Listovsky T, Oren YS, Yudkovsky Y, Mahbubani HM, Weiss AM, et al. 2004. Mammalian Cdh1/Fzr mediates its own degradation. EMBO 7. 23:1619-26

82. Listovsky T, Sale JE. 2013. Sequestration of CDH1 by MAD2L2 prevents premature APC/C activation prior to anaphase onset. F. Cell Biol. 203:87-100

83. Liu D, Vader G, Vromans MJ, Lampson MA, Lens SM. 2009. Sensing chromosome bi-orientation by spatial separation of aurora B kinase from kinetochore substrates. Science 323:1350-53

84. Liu ST, Chan GK, Hittle JC, Fujii G, Lees E, Yen TJ. 2003. Human MPS1 kinase is required for mitotic arrest induced by the loss of CENP-E from kinetochores. Mol. Biol. Cell 14:1638-51

85. Luo X, Tang Z, Rizo J, Yu H. 2002. The Mad2 spindle checkpoint protein undergoes similar major conformational changes upon binding to either Mad1 or Cdc20. Mol. Cell 9:59-71

86. Luo X, Tang Z, Xia G, Wassmann K, Matsumoto T, et al. 2004. The Mad2 spindle checkpoint protein has two distinct natively folded states. Nat. Struct. Mol. Biol. 11:338-45

87. Machida YJ, Dutta A. 2007. The APC/C inhibitor, Emi1, is essential for prevention of rereplication. Genes Dev. 21:184-94

88. Maciejowski J, George KA, Terret ME, Zhang C, Shokat KM, Jallepalli PV. 2010. Mps1 directs the assembly of Cdc20 inhibitory complexes during interphase and mitosis to control $\mathrm{M}$ phase timing and spindle checkpoint signaling. F. Cell Biol. 190:89-100

89. Malureanu LA, Jeganathan KB, Hamada M, Wasilewski L, Davenport J, van Deursen JM. 2009. BubR1 $\mathrm{N}$ terminus acts as a soluble inhibitor of cyclin B degradation by APC/C(Cdc20) in interphase. Dev. Cell 16:118-31

90. Margottin-Goguet F, Hsu JY, Loktev A, Hsieh HM, Reimann JD, Jackson PK. 2003. Prophase destruction of Emil by the SCF( $\beta$ TrCP/Slimb) ubiquitin ligase activates the anaphase promoting complex to allow progression beyond prometaphase. Dev. Cell 4:813-26

91. Matsumoto ML, Wickliffe KE, Dong KC, Yu C, Bosanac I, et al. 2010. K11-linked polyubiquitination in cell cycle control revealed by a K11 linkage-specific antibody. Mol. Cell 39:477-84

92. Meraldi P, Draviam VM, Sorger PK. 2004. Timing and checkpoints in the regulation of mitotic progression. Dev. Cell 7:45-60

93. Meyer HJ, Rape M. 2014. Enhanced protein degradation by branched ubiquitin chains. Cell 157:910-21

94. Miller JJ, Summers MK, Hansen DV, Nachury MV, Lehman NL, et al. 2006. Emi1 stably binds and inhibits the anaphase-promoting complex/cyclosome as a pseudosubstrate inhibitor. Genes Dev. 20:2410 20

95. Murray AW, Solomon MJ, Kirschner MW. 1989. The role of cyclin synthesis and degradation in the control of maturation promoting factor activity. Nature 339:280-86 
96. Musacchio A, Salmon ED. 2007. The spindle-assembly checkpoint in space and time. Nat. Rev. Mol. Cell Biol. 8:379-93

97. Nakayama KI, Nakayama K. 2006. Ubiquitin ligases: cell-cycle control and cancer. Nat. Rev. Cancer 6:369-81

98. Nam HJ, Naylor RM, van Deursen JM. 2015. Centrosome dynamics as a source of chromosomal instability. Trends Cell Biol. 25:65-73

99. Nam HJ, van Deursen JM. 2014. Cyclin B2 and p53 control proper timing of centrosome separation. Nat. Cell Biol. 16:538-49

100. Naylor RM, Jeganathan KB, Cao X, van Deursen JM. 2016. Nuclear pore protein NUP88 activates anaphase-promoting complex to promote aneuploidy. F. Clin. Investig. 126:543-59

101. Orr B, Compton DA. 2013. A double-edged sword: how oncogenes and tumor suppressor genes can contribute to chromosomal instability. Front. Oncol. 3:164

102. Park I, Lee HO, Choi E, Lee YK, Kwon MS, et al. 2013. Loss of BubR1 acetylation causes defects in spindle assembly checkpoint signaling and promotes tumor formation. F. Cell Biol. 202:295-309

103. Pfau SJ, Amon A. 2012. Chromosomal instability and aneuploidy in cancer: from yeast to man. $E M B O$ Rep. 13:515-27

104. Prinz S, Hwang ES, Visintin R, Amon A. 1998. The regulation of Cdc20 proteolysis reveals a role for APC components Cdc23 and Cdc27 during S phase and early mitosis. Curr. Biol. 8:750-60

105. Rahmani Z, Gagou ME, Lefebvre C, Emre D, Karess RE. 2009. Separating the spindle, checkpoint, and timer functions of BubR1.7. Cell Biol. 187:597-605

106. Rape M, Kirschner MW. 2004. Autonomous regulation of the anaphase-promoting complex couples mitosis to S-phase entry. Nature 432:588-95

107. Reddy SK, Rape M, Margansky WA, Kirschner MW. 2007. Ubiquitination by the anaphase-promoting complex drives spindle checkpoint inactivation. Nature 446:921-25

108. Reimann JD, Gardner BE, Margottin-Goguet F, Jackson PK. 2001. Emi1 regulates the anaphasepromoting complex by a different mechanism than Mad2 proteins. Genes Dev. 15:3278-85

109. Ricke RM, van Deursen JM. 2013. Aneuploidy in health, disease, and aging. F. Cell Biol. 201:11-21

110. Ricke RM, van Ree JH, van Deursen JM. 2008. Whole chromosome instability and cancer: a complex relationship. Trends Genet. 24:457-66

111. Rodriguez-Bravo V, Maciejowski J, Corona J, Buch HK, Collin P, et al. 2014. Nuclear pores protect genome integrity by assembling a premitotic and Mad1-dependent anaphase inhibitor. Cell 156:1017-31

112. Santaguida S, Amon A. 2015. Short- and long-term effects of chromosome mis-segregation and aneuploidy. Nat. Rev. Mol. Cell Biol. 16:473-85

113. Shah JV, Botvinick E, Bonday Z, Furnari F, Berns M, Cleveland DW. 2004. Dynamics of centromere and kinetochore proteins: implications for checkpoint signaling and silencing. Curr. Biol. 14:942-52

114. Shindo N, Kumada K, Hirota T. 2012. Separase sensor reveals dual roles for separase coordinating cohesin cleavage and Cdk1 inhibition. Dev. Cell 23:112-23

115. Silk AD, Zasadil LM, Holland AJ, Vitre B, Cleveland DW, Weaver BA. 2013. Chromosome missegregation rate predicts whether aneuploidy will promote or suppress tumors. PNAS 110:E4134-41

116. Silkworth WT, Cimini D. 2012. Transient defects of mitotic spindle geometry and chromosome segregation errors. Cell Div. 7:19

117. Silkworth WT, Nardi IK, Paul R, Mogilner A, Cimini D. 2012. Timing of centrosome separation is important for accurate chromosome segregation. Mol. Biol. Cell 23:401-11

118. Sivakumar S, Gorbsky GJ. 2015. Spatiotemporal regulation of the anaphase-promoting complex in mitosis. Nat. Rev. Mol. Cell Biol. 16:82-94

119. Skoufias DA, Andreassen PR, Lacroix FB, Wilson L, Margolis RL. 2001. Mammalian mad2 and bub1/bubR1 recognize distinct spindle-attachment and kinetochore-tension checkpoints. PNAS 98:4492-97

120. Stegmeier F, Rape M, Draviam VM, Nalepa G, Sowa ME, et al. 2007. Anaphase initiation is regulated by antagonistic ubiquitination and deubiquitination activities. Nature 446:876-81

121. Sudakin V, Chan GK, Yen TJ. 2001. Checkpoint inhibition of the APC/C in HeLa cells is mediated by a complex of BUBR1, BUB3, CDC20, and MAD2. 7. Cell Biol. 154:925-36 
122. Tanaka TU, Rachidi N, Janke C, Pereira G, Galova M, et al. 2002. Evidence that the Ipl1-Sli15 (Aurora kinase-INCENP) complex promotes chromosome bi-orientation by altering kinetochore-spindle pole connections. Cell 108:317-29

123. Taylor SS, Ha E, McKeon F. 1998. The human homologue of Bub3 is required for kinetochore localization of Bub1 and a Mad3/Bub1-related protein kinase. F. Cell Biol. 142:1-11

124. Taylor SS, McKeon F. 1997. Kinetochore localization of murine Bub1 is required for normal mitotic timing and checkpoint response to spindle damage. Cell 89:727-35

125. Thompson SL, Compton DA. 2008. Examining the link between chromosomal instability and aneuploidy in human cells. 7. Cell Biol. 180:665-72

126. Thompson SL, Compton DA. 2010. Proliferation of aneuploid human cells is limited by a p53-dependent mechanism. 7. Cell Biol. 188:369-81

127. Thompson SL, Compton DA. 2011. Chromosome missegregation in human cells arises through specific types of kinetochore-microtubule attachment errors. PNAS 108:17974-78

128. Uhlmann F, Lottspeich F, Nasmyth K. 1999. Sister-chromatid separation at anaphase onset is promoted by cleavage of the cohesin subunit Scc1. Nature 400:37-42

129. Visintin R, Craig K, Hwang ES, Prinz S, Tyers M, Amon A. 1998. The phosphatase Cdc14 triggers mitotic exit by reversal of Cdk-dependent phosphorylation. Mol. Cell 2:709-18

130. Vitale I, Galluzzi L, Castedo M, Kroemer G. 2011. Mitotic catastrophe: a mechanism for avoiding genomic instability. Nat. Rev. Mol. Cell Biol. 12:385-92

131. Vitre BD, Cleveland DW. 2012. Centrosomes, chromosome instability (CIN) and aneuploidy. Curr. Opin. Cell Biol. 24:809-15

132. Waizenegger I, Gimenez-Abian JF, Wernic D, Peters JM. 2002. Regulation of human separase by securin binding and autocleavage. Curr. Biol. 12:1368-78

133. Waizenegger IC, Hauf S, Meinke A, Peters JM. 2000. Two distinct pathways remove mammalian cohesin from chromosome arms in prophase and from centromeres in anaphase. Cell 103:399-410

134. Walczak CE, Cai S, Khodjakov A. 2010. Mechanisms of chromosome behaviour during mitosis. Nat. Rev. Mol. Cell Biol. 11:91-102

135. Wang W, Kirschner MW. 2013. Emi1 preferentially inhibits ubiquitin chain elongation by the anaphasepromoting complex. Nat. Cell Biol. 15:797-806

136. Wang Y, Waters J, Leung ML, Unruh A, Roh W, et al. 2014. Clonal evolution in breast cancer revealed by single nucleus genome sequencing. Nature 512:155-60

137. Waters JC, Chen RH, Murray AW, Salmon ED. 1998. Localization of Mad2 to kinetochores depends on microtubule attachment, not tension. F. Cell Biol. 141:1181-91

138. Weaver BA, Silk AD, Montagna C, Verdier-Pinard P, Cleveland DW. 2007. Aneuploidy acts both oncogenically and as a tumor suppressor. Cancer Cell 11:25-36

139. Welburn JP, Vleugel M, Liu D, Yates JR 3rd, Lampson MA, et al. 2010. Aurora B phosphorylates spatially distinct targets to differentially regulate the kinetochore-microtubule interface. Mol. Cell 38:383-92

140. Williams BR, Prabhu VR, Hunter KE, Glazier CM, Whittaker CA, et al. 2008. Aneuploidy affects proliferation and spontaneous immortalization in mammalian cells. Science 322:703-9

141. Williamson A, Banerjee S, Zhu X, Philipp I, Iavarone AT, Rape M. 2011. Regulation of ubiquitin chain initiation to control the timing of substrate degradation. Mol. Cell 42:744-57

142. Xia G, Luo X, Habu T, Rizo J, Matsumoto T, Yu H. 2004. Conformation-specific binding of p31(comet) antagonizes the function of Mad2 in the spindle checkpoint. EMBO 7. 23:3133-43

143. Yang F, Huang Y, Dai W. 2012. Sumoylated BubR1 plays an important role in chromosome segregation and mitotic timing. Cell Cycle 11:797-806

144. Yang M, Li B, Tomchick DR, Machius M, Rizo J, et al. 2007. p31 comet blocks Mad2 activation through structural mimicry. Cell 131:744-55

145. Yang Z, Kenny AE, Brito DA, Rieder CL. 2009. Cells satisfy the mitotic checkpoint in Taxol, and do so faster in concentrations that stabilize syntelic attachments. F. Cell Biol. 186:675-84

146. Zachariae W, Schwab M, Nasmyth K, Seufert W. 1998. Control of cyclin ubiquitination by CDKregulated binding of Hctl to the anaphase promoting complex. Science 282:1721-24 
147. Zeng X, Sigoillot F, Gaur S, Choi S, Pfaff KL, et al. 2010. Pharmacologic inhibition of the anaphasepromoting complex induces a spindle checkpoint-dependent mitotic arrest in the absence of spindle damage. Cancer Cell 18:382-95

148. Zhang Y, Foreman O, Wigle DA, Kosari F, Vasmatzis G, et al. 2012. USP44 regulates centrosome positioning to prevent aneuploidy and suppress tumorigenesis. F. Clin. Investig. 122:4362-74

149. Zou H, McGarry TJ, Bernal T, Kirschner MW. 1999. Identification of a vertebrate sister-chromatid separation inhibitor involved in transformation and tumorigenesis. Science 285:418-22 


\section{Contents}

A Life Investigating Pathways that Repair Broken Chromosomes

Fames E. Haber....

Sex-Biased Gene Expression

Sonja Grath and Fohn Parsch ....

Aneuploidy in Cancer and Aging

Ryan M. Naylor and Fan M. van Deursen

Transition Metals and Virulence in Bacteria

Lauren D. Palmer and Eric P. Skaar....

Sperm Meets Egg: The Genetics of Mammalian Fertilization

Enrica Bianchi and Gavin 7. Wright

Robust Yet Fragile: Expression Noise, Protein Misfolding, and Gene

Dosage in the Evolution of Genomes

7. Chris Pires and Gavin C. Conant

Evolution in the Cycles of Life

Fobn L. Bowman, Keiko Sakakibara, Chibiro Furumizu, and Tom Dierschke....

Functions, Regulation, and Therapeutic Implications of the ATR

Checkpoint Pathway

Stephanie A. Yazinski and Lee Zou

Control of Meiotic Crossovers: From Double-Stand Break Formation

to Designation

Stephen Gray and Paula E. Cohen

The Plant Microbiota: Systems-Level Insights and Perspectives

Daniel B. Muiller, Christine Vogel, Yang Bai, and Fulia A. Vorbolt

Genome-Wide Analysis of RNA Secondary Structure

Philip C. Bevilacqua, Laura E. Ritchey, Zhao Su, and Sarab M. Assmann

Single-Cell and Single-Molecule Analysis of Gene Expression Regulation

Maria Vera, Jeetayu Biswas, Adrien Senecal, Robert H. Singer, and Hye Yoon Park .. 267

Conservation and Variability of Meiosis Across the Eukaryotes Fosef Loidl .....

Monoallelic Gene Expression in Mammals

Andrew Chess ....
Annual Review of Genetics

Volume 50, 2016 
Proteopathic Strains and the Heterogeneity of Neurodegenerative Diseases

Lary C. Walker

The Ecology and Evolution of Cancer: The Ultra-Microevolutionary Process

Chung-I Wu, Hurng-Yi Wang, Shaoping Ling, and Xuemei Lu

Regulation and Role of Fungal Secondary Metabolites

Juliane Macheleidt, Derek F. Mattern, Juliane Fischer, Tina Netzker,

Jakob Weber, Volker Schroeckh, Vito Valiante, and Axel A. Brakhage

Eukaryotic DNA Polymerases in Homologous Recombination

Mitch McVey, Varandt Y. Khodaverdian, Damon Meyer,

Paula Gonçalves Cerqueira, and Wolf-Dietrich Heyer

Regulated Proteolysis in Bacteria: Caulobacter

Kamal Kishore Foshi and Peter Chien

Mosquito Vectors and the Globalization of Plasmodium falciparum Malaria

Alvaro Molina-Cruz, Martine M. Zilversmit, Daniel E. Neafsey,

Daniel L. Hartl, and Carolina Barillas-Mury

Plant Transgenerational Epigenetics

Leandro Quadrana and Vincent Colot

The Genetics of Enteropathogenic Escherichia coli Virulence

Jaclyn S. Pearson, Cristina Giogha, Tania Wong Fok Lung, and Elizabeth L. Hartland....

Deciphering Combinatorial Genetics

Alan S.L. Wong, Gigi C.G. Choi, and Timotby K. Lu

Interorgan Communication Pathways in Physiology: Focus on

Drosophila

Ilia A. Droujinine and Norbert Perrimon

Cell-Specific Targeting of Genetically Encoded Tools for

Neuroscience

Lucas Sjulson, Daniela Cassataro, Shamik DasGupta, and Gero Miesenböck

Vaccination via Chloroplast Genetics: Affordable Protein Drugs for the Prevention and Treatment of Inherited or Infectious Human Diseases

Henry Daniell, Hui-Ting Chan, and Elise K. Pasoreck.....

\section{Errata}

An online log of corrections to Annual Review of Genetics articles may be found at http://www.annualreviews.org/errata/genet 


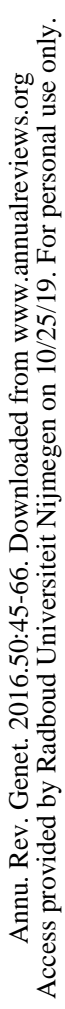

